\title{
Expanding Space: the Root of all Evil?
}

\author{
Matthew J. Francis ${ }^{\mathrm{A}, \mathrm{D}}$, Luke A. Barnes ${ }^{\mathrm{A}, \mathrm{B}}$, J. Berian James ${ }^{\mathrm{A}, \mathrm{C}}$, and Geraint F. Lewis ${ }^{\mathrm{A}}$ \\ A Institute of Astronomy, School of Physics, University of Sydney, NSW 2006, Australia \\ ${ }^{B}$ Institute of Astronomy, Madingley Rd, Cambridge, UK \\ ${ }^{C}$ Institute for Astronomy, Blackford Hill, Edinburgh EH9 3HJ, UK \\ D Corresponding author. Email: mfrancis@physics.usyd.edu.au
}

Received 2007 June 1, accepted 2007 June 29

\begin{abstract}
While it remains the staple of virtually all cosmological teaching, the concept of expanding space in explaining the increasing separation of galaxies has recently come under fire as a dangerous idea whose application leads to the development of confusion and the establishment of misconceptions. In this paper we develop a notion of expanding space that is completely valid as a framework for the description of the evolution of the universe and whose application allows an intuitive understanding of the influence of universal expansion. We also demonstrate how arguments against the concept in general have failed thus far, as they imbue expanding space with physical properties not consistent with the expectations of general relativity.
\end{abstract}

Keywords: cosmology: theory

\section{Introduction}

When the mathematical picture of cosmology is first introduced to students in senior undergraduate or junior postgraduate courses, a key concept to be grasped is the relation between the observation of the redshift of galaxies and the general relativistic picture of the expansion of the Universe. When presenting these new ideas, lecturers and textbooks often resort to analogies of stretching rubber sheets or cooking raisin bread to allow students to visualise how galaxies are moved apart, and waves of light are stretched by the 'expansion of space'. These kinds of analogies are apparently thought to be useful in giving students a mental picture of cosmology, before they have the ability to directly comprehend the implications of the formal general relativistic description. However, the academic argument surrounding the expansion of space is not as clear as standard explanations suggest; an interested student and reader of New Scientist may have seen Rees \& Weinberg (1993) state

...how is it possible for space, which is utterly empty, to expand? How can nothing expand? The answer is: space does not expand. Cosmologists sometimes talk about expanding space, but they should know better.

while being told by Harrison (2000) that

expansion redshifts are produced by the expansion of space between bodies that are stationary in space.

What is a lay-person or proto-cosmologist to make of this apparently contradictory situation?

Whether or not attempting to describe the observations of the cosmos in terms of expanding space is a useful goal, regardless of the devices used to do so, is far from uncontroversial. Recent attacks on the physical concept of expanding space have centred on the motion of test particles in the expanding universe. Whiting (2004), Peacock ${ }^{1}$ and others claim that expanding space fails to adequately explain the motion of test particles and hence that it should be abandoned. But what, exactly, is at fault? Crucially, these claims rely on falsifying predictions made from using expanding space as a tool to guide intuition, to bypass the full mathematical calculation. However, the very meaning of the phrase expanding space is not rigorously defined, despite its widespread use in teaching and textbooks. Hence, it is prudent to be wary of predictions based on such a poorly defined intuitive frameworks.

In recent work, Barnes et al. (2006) solved the testparticle motion problem for universes with arbitrary asymptotic equation of state $w$ and found agreement between the general relativistic solution and the expected behaviour of particles in expanding space. We suggest that the apparent conflict between this work and others, for instance Chodorowski (2006b), lies predominantly in differing meanings of the very concept of expanding space. This is unsurprising, given that it is a phrase and concept often stated but seldom defined with any rigour.

In this paper, we examine the picture of expanding space within the framework of fully general relativistic cosmologies and develop it into a precise definition for understanding the dynamical properties of FriedmanRobertson-Walker (FRW) spacetimes. This framework is pedagogically superior to ostensibly simpler misleading formulations of expanding space - or more general schemes to picture the expansion of the Universe - such

\footnotetext{
${ }^{1}$ www.roe.ac.uk/ jap/book/additions.html
} 
as kinematic models and approximations to special relativity or Newtonian mechanics, since it is both clearer and easier to understand as well as being a more accurate approximation. In particular, it must be emphasised that the expansion of space does not, in and of itself, represent new physics that is a cause of observable effects, such as redshift. Rather the expansion of space is an intuitive framework for understanding the effects of General Relativity.

In Sections 2.1 to 2.4 we detail this working picture of expanding space, before Section 2.5 explores this definition in terms of the motion of free particles in an expanding universe. The application of this approach to dispel arguments against the expansion space is demonstrated in Section 2.6, before the conclusions are presented in Section 3.

\section{Expanding Space}

In understanding the concept of expanding space, it is important to examine the basic premise of general relativity, neatly packaged in John Wheeler's adage

matter tells Spacetime how to curve, and Spacetime tells matter how to move

which sets out the dynamical relationship between the geometry of spacetime and the density and pressures of fluids contained therein.

However, if the prominent cosmologists quoted in the previous section, will ask 'how can space, which is ultimately empty, expand', we must also ask the question of how this 'nothingness' of the vacuum can be curved? By reducing Wheeler's adage to

matter tells matter how to move

the concept of spacetime, just like the aether, can be banished as being non-existent and unnecessary (e.g. Chodorowski 2006b). Such a picture is not as heretical as it seems: Weinberg (1972), in his classic text on general relativity, questions the whole geometric picture of relativity, and the language it encompasses, as an unfortunate hangover which is not necessary.

It is enlightening to realise that this situation occurs in many branches of physics. For example, in terms analogous to Wheeler's adage, electromagnetism can be reduced to

\section{charges tell charges how to move}

but the employed framework contains the concepts of electric and magnetic fields which are as intrinsically unobservable as spacetime. Furthermore, these fields obey strict mathematical relationships, through the equations of Maxwell, and many researchers can picture the evolution of these fields in dynamical circumstances, even though it is just charges telling charges what to do.

Hence, we arrive at the view point that while general relativity is just 'matter telling matter how to move', its framework contains deformable and stretchy spacetime. As with electromagnetism, this field is not intrinsically detectable, but does obey strict mathematical relations. Similarly, as correct as it is to think of electromagnetism in terms of electric and magnetic fields, we can think of general relativity in terms of the dynamical entity of spacetime as long as we develop our intuition in terms of the underlying mathematics, and not try to match the properties of spacetime to the properties of dough or rubber; just as it would make no sense to attempt to construct a physical or thought experiment to attempt to prove or disprove the real existence of magnetic fields, it is similarly meaningless to discuss the expansion of space in these terms.

To illustrate how short this pragmatic formalism falls of being platitude, one need look no further than Abramowicz et al. (2006), in which a thought experiment of laser ranging in an FRW Universe is proposed to 'prove' that space must expand. This is sensibly refuted by Chodorowski (2006b), but followed by a spurious counter-claim that such a refutation likewise proves space does not expand. The exercise is futile: What matters on a technical level are predictions for observable quantities, which of course are the same regardless of how the problem is pictured and what co-ordinate system is chosen. The expansion of space is no more extant than magnetic fields are, and exists only as a tool for understanding the unambiguous predictions of GR, not a force-like term in a dynamical equation. A recent example of the dangers of thinking of expanding space as a real physical theory is contained in Table 2 of Lieu (2007) in which the expansion of space is lumped together with the Big Bang, Dark Energy, Dark Matter and Inflation as a physical theory demanding verification.

We can certainly agree that this kind of misuse of the term 'expansion of space' is fallacious and most certainly dangerous. But throwing the baby of an intuitive framework out with the bathwater of misconceptions leaves us only with bare mathematics, which in the case of general relativity is particularly daunting for the uninitiated, and useless as a conceptual device.

\subsection{The Cosmological Picture}

We turn now to outlining the way in which the expansion of space can be retained as a useful pedagogical device, while avoiding the pitfalls of misleading formulations. It is worth starting from first principles and asking what the general relativistic picture of cosmology actually contains. The adoption of the cosmological principle, in that the Universe is homogeneous and isotropic, restricts the form of the underlying geometry of the Universe, expressed in terms of the FRW metric. With this metric, the continuity equation demonstrates that in other than finely-tuned or contrived examples, the density and pressures of cosmological fluids must change over cosmic time, and it is this change that represents the basic property of an expanding (or contracting) universe.

The general-relativistic picture also allows the definition of privileged, co-moving observers (said to reside in the Hubble flow) within the expanding universe, those at 
rest with respect to the cosmological fluids. In our Universe we know we are not one of these privileged observers as our measured CMB dipole reveals our peculiar motion with respect to the background photons. Being at rest with regards to the cosmic fluid, the proper time for these privileged observers ticks at the same rate as cosmic time and hence the watches of all privileged observers are synchronised. In an expanding universe, the change of the metric implies that the physical distance between any two privileged observers increases with time, and consequently, if eight of these co-moving observers are used to define the corners of a cube, the volume of the cube must increase with time.

Remembering that the FRW metric describes a homogeneous universe filled with a fluid of uniform density, and assuming that test observers can measure their velocity with respect to that fluid, we can now describe the formal statement of the phenomenon we refer to as expanding space:

The distance between observers at rest with respect to the cosmic fluid increases with time.

Since two bodies, both at rest with respect to the fluid defining the FRW metric, find the distance between them has increased after a certain time interval, it seems sensible to suggest that there is more space between them than there was previously. It may be misleading to suggest that the space that was there stretched itself as the universe expanded. Perhaps a better description, in simple terms, is to suggest that more space appeared, or 'welled up' between the two observers, however this is a largely semantic distinction.

We are also in a good position to understand why the expansion can be thought of locally in kinematical, even Newtonian terms. We can imagine attaching a Minkowski frame to each point in the Hubble flow. The local cosmological fluid is stationary with respect to this frame. Whilst only perfectly accurate in an infinitesimally small region, the Minkowski frame can be used as an approximation for regions much smaller than the Hubble radius. The Hubble flow is then viewed as a purely kinematical phenomenon - objects recede because they have been given an initial velocity proportional to distance. This does not argue against expanding space: the equivalence principle guarantees that any free-falling observer in any GR spacetime can use SR locally. $^{2}$

\footnotetext{
2 The kinematical view can be useful, but remains only a local approximation. The exception is the Milne model: in an empty universe we can make a coordinate transformation that exchanges the FRW metric for the Minkowski metric (see Harrison 2000, p. 88), effectively extending our local Minkowski frame to all spacetime. This is only possible because there is no cosmological fluid to define the rest frame of the universe. Hence the Milne model cannot be used to make general comments on the nature of the cosmological expansion, cf. Chodorowski (2006a). Recently it has been claimed by Chodorowski (2006b) that conformal transformations of general FRW metrics can produce a common global frame describing the entire spacetime, analogous to the common Minkowski frame in the Milne model. This will be examined in a future contribution.
}

\subsection{Local Expansions}

At the global level, Peacock ${ }^{1}$ suggests that the expansion of space is uncontroversial since

the total volume of a closed universe is a well-defined quantity that increases with time, so of course space is expanding

\section{but questions whether}

this concept has a meaningful local counterpart?... Is the space in my bedroom expanding, and what would this mean?

Retaining the relativistic picture of expanding space, it is easy to address the question of what happens to Peacock's bedroom, namely it will evolve as determined by the relativistic equations. But as ever, knowledge of the scenario, and particularly the initial conditions, is vital; the walls of the bedroom are held together by electromagnetic forces and hence are not following geodesics, and the distribution of matter has collapsed and is not uniform, and so the underlying geometry of spacetime in this region needs to be calculated; it would not be represented by the FRW spacetime of the homogeneous and isotropic universe. Clearly, if the universe were homogeneous on scales smaller than Peacock's bedroom, and the walls were not held together by electromagnetic or other forces, and the particles making up the wall were at rest with the cosmological fluid which, importantly, requires that they not be initially at rest with respect to one another, then indeed as the universe expands the total volume of the bedroom would increase. The many conditions listed above are (at least approximately) true for galaxies not bound in common groups and hence they behave in ways that can be understood and predicted via the framework of expanding space.

This leads to an important point, namely that we should not expect the global behaviour of a perfectly homogeneous and isotropic model to be applicable when these conditions are not even approximately met. The expansion of space fails to have a 'meaningful local counterpart', not because there is some sleight of hand involved in considering the two regimes, but because the physical conditions that manifest the effects described as the expansion of space are not met in the average suburban bedroom.

\subsection{Dark Energy}

In a matter-dominated universe, the statement in the preceding section regarding the metric in the region of a collapsed object being unlike the FRW metric is straightforward. However, if the universe is dominated (as we believe ours currently is) by an energy that by definition is homogeneous, or only inhomogeneous on very large scales, then we must be more careful. In this case the dominant driver of the specifics of the expansion rate will apply equally on all scales. However, so long as the equation of state $w$ of the dark energy obeys the condition $w \geq-1$ the energy density will not increase with time and bound 
structures will remain bound and stable. Effectively the region of spacetime inside a bound structure will in fact be matter-dominated, even though the global mean density is dark energy-dominated.

\subsection{The Value of Analogies}

What efficacy then, if any, do the common expanding universe analogies have? The balloon-with-dots or bread-with-raisins analogies, like any analogies, are useful so long as we are aware of what they successfully illustrate and what constitutes pushing the analogy too far. They show how a homogeneous expansion inevitably results in velocity being proportional to distance, and also gives an intuition for how the expansion of the universe looks the same from every point in the universe. They illustrate that the universe does not expand into previously existing empty space; it consists of expanding space. But using these analogies to visualise a mechanism like a frictional or viscous force is taking the analogy too far. They correctly demonstrate the effects of the expansion of the universe, but not the mechanism. That they fail at some level is hardly surprising: we're representing 4-dimensional pseudo-Riemannian manifolds with party supplies. We can't manipulate frames like gravity can.

\subsection{The Challenge of Particle Motion}

We now turn to the issue of test particle motion, since this is at the heart of many of the attacks on expanding space. The classic thought experiment used is the "tethered galaxy' problem (Harrison 1995). In this, a test galaxy in an expanding universe is held at rest with respect to the origin at a cosmological distance. By Hubble's law we would expect this galaxy to be receding, however we prevent this, artificially holding the test galaxy in place. The question is, when the galaxy is released, what does it do? Since critics of the expanding space concept argue that the Newtonian analogue of expanding space is the presence of some kind of viscous force, dragging the galaxies apart like objects carried along by a river, therefore in this thought experiment, the test particle should pick up a velocity away from the origin due to the expanding river of space. In fact, what the particle does once being released depends on the acceleration of the universe. If the scale factor is decelerating the particle moves towards the origin, rather than away. ${ }^{3}$ That acceleration is important in the question of the future velocity of a particle is a concept that a student of the most elementary physics is comfortable with. Since expanding space has apparently mislead our intuition so severely this appears to demonstrate the dangers of this interpretation. But is this a fair test of expanding space? If the balloon or baking-bread analogy is used to attempt to picture this situation then indeed the incorrect

\footnotetext{
${ }^{3}$ The case where the universe is accelerating is a little more complex. For small initial distances the particle will move away from the origin as might be expected. However for greater distances the particle can initially move towards the origin. The reasons are somewhat subtle and we will focus on the accelerating case for clarity. See Grøn \& Elgarøy (2006) for full details of the accelerating universe case.
}

answer is easily reached. However, as mentioned, this is pushing these analogies too far, since they are useful in picturing what an expanding universe looks like, but do not speak of what drives that expansion.

This is the central issue and point of confusion. Galaxies move apart because they did in the past, causing the density of the Universe to change and therefore altering the metric of spacetime. We can describe this alteration as the expansion of space, but the key point is that it is a result of the change in the mean energy density, not the other way around. The expansion of space does not cause the distance between galaxies to increase, rather this increase in distance causes space to expand, or more plainly that this increase in distance is described by the framework of expanding space. There is therefore no need to look for Newtonian analogues to the expansion of space, since it is an effect rather than a cause. In any case, why should we be seeking Newtonian analogues when we know general relativity describes the situation well, can be described in simple terms and any Newtonian view will break down at at a non-trivial limit? Whiting (2004) describes the tethered galaxy problem in Newtonian terms and uses Newtonian equations to make predictions about the asymptotic behaviour of the test particle. However, as shown in Barnes et al. (2006) these equations deviate significantly from the general relativistic results which begs the question of why Newtonian analogues should be sought for fundamentally relativistic problems?

Can the tethered galaxy problem by understood in the context of expanding space? We contend that the answer is yes. The key is to carefully examine the initial conditions of the particle. Whiting (2004) and Peacock ${ }^{1}$ have in mind that the initial conditions of the problem describe a particle dropped innocently into the universe. It has no proper velocity and thus no prejudice: It is free to go wherever expanding space wishes to take it. This is certainly true from a kinematic, Newtonian perspective. The particle is at rest in our chosen inertial frame and approaches the origin due to the gravitational attraction of the matter between the particle and the origin. This is locally valid and even useful, but it is not how to understand the scenario from an expanding space perspective. The motion of the particle must be analysed with respect to its local rest frame of the test particles, provided by the Hubble flow. In this frame, we see the original observer moving at $v_{\mathrm{rec}, 0}$ and the particle shot out of the local Hubble frame at $v_{\text {pec, } 0}$, so that the scenario resembles a race. Since their velocities are initially equal, the winner of the race is decided by how these velocities change with time. In a decelerating universe, the recession velocity of the original observer decreases, handing victory to the test particle, which catches up with the observer.

The difference between the kinematic and expanding space interpretation is well illustrated by figure 1 of Davis, Lineweaver, \& Webb (2001). Their figure 1a shows the kinematic perspective: the observer and the tethered particle are at rest with respect to each other and gravitational attraction will bring them together. Their figure $1 \mathrm{~b}$ shows 
the scenario as seen from the local rest frame of the tethered particle, i.e. a race between the original observer and the test particle. The original observer should view the initial conditions of the test particle, not as neutral, but as a battle between motion through space and the expansion of space. The expansion of space has been momentarily nullified by the initial conditions, so we must ask how the expansion of space changes with time.

We contend that this explanation successfully incorporates test particle motion into the concept of expanding space. In particular, it shows why it is wrong to expect, on the basis of the balloon analogy, that expanding space would carry the particle away. The alternative is either to give up on a physical concept entirely, so that the only rationale for the cosmological facts is that 'that's what the maths tells us', or to formulate a new framework into which these facts and more can be accommodated. The first option is unsavoury, the second unlikely, unless one wants to discard GR entirely and formulate cosmology using only Newtonian ideas (Tipler 1996).

\subsection{Using Expanding Space}

In this section, we examine in detail the employment of the concept of expanding space in a number of cosmological scenarios.

\subsubsection{Superluminal Recession Velocities}

By failing to place a limit on the range of validity of Hubble's Law, the FRW metric implies that there is no speed limit on recession velocities, seeming to violate a fundamental principle of relativity by implying superluminal motion. This is a frequent cause of concern and confusion. In terms of the proper distance $D$ defined as $D=a \chi$ and the cosmic time $t$ in the FRW metric then the differential $\mathrm{d} D / \mathrm{d} t$ most certainly can exceed unity, and hence by this definition of velocity, represents superluminal motion. However, as shown in Grøn \& Elgarøy (2006) and Page (1993) we can, by a co-ordinate transformation, describe simple Minkowski space-time as the FRW metric of an empty universe, known as the Milne model. In the Minkowski special relativistic co-ordinates we of course cannot have superluminal motion. However in the new Milne model co-ordinates we do find that $\mathrm{d} D / \mathrm{d} t>1$ beyond a certain distance from the origin. Thus we have apparently described superluminal motion in a spacetime that we know cannot permit such a phenomenon. Via conformal transformations, it is possible (see e.g. Chodorowski 2006b) to make a similar transformation between general FRW metrics and conformally related Minkowskilike metrics. Again in the FRW case $\mathrm{d} D / \mathrm{d} t$ make exceed unity, while in the conformal co-ordinates the speed is limited. While some authors (e.g. Chodorowski 2006b and Page 1993) have argued that this demonstrates that superluminal recession is impossible, others, for instance Grøn \& Elgarøy (2006) have argued that superluminal recession is a fundamental consequence of the FRW metric. As pointed out in Barnes et al. (2006), many of the debates surrounding expanding space turn out to be based on different definitions of poorly defined concepts, in this case the term superluminal. If we mean by superluminal that the motion described in the co-ordinates of the Minkowski (or conformal Minkowski-like) frame defined by extending the local inertial frame of an given observer is greater than unity then everyone agrees that this does not occur. On the other hand, if we take the FRW co-ordinates it is clear that there is no limit on the recession velocity: if we choose to call this superluminal motion, then it indeed occurs. The debate seems to boil down to whether this should or should not be given the name 'superluminal' but crucially the physical predictions made by either camp will be identical. What matters is not what we call the phenomenon but whether the understanding an individual has of a given term reflects reality and it is clear that not all the authors mentioned above held common meanings of the term superluminal.

What does matter is that we have a framework for understanding the consequences of the FRW metric that is unambiguous and easy to understand. In the seminal work of Davis \& Lineweaver (2004) several common mistakes regarding recession velocities are examined. The authors take a strong view that recession velocities really are superluminal but more importantly show the types of mistakes that can be made by on the one hand using the FRW formalism and on the other hand making ad hoc 'corrections' to prevent apparent superluminal motion in the FRW co-ordinates. The key point to take from this is that one must be consistent; if we use the very convenient FRW metric we must be aware that the recession speed is not limited in any way. If this is uncomfortable alternative co-ordinates may be adopted and, if used consistently, will return the same physical predictions as the correctly used FRW co-ordinates.

We will now outline how velocities can be treated consistently and clearly within the framework of expanding space. Consider a test particle moving radially with coordinate velocity $\dot{\chi}(t)$. The proper velocity of the object as measured by an observer at the origin is:

$$
\dot{r}_{\mathrm{p}}=\dot{R}(t) \chi(t)+R(t) \dot{\chi}(t)
$$

The first term is the same as for a particle in the Hubble flow at the same co-moving coordinate and depends on the rate of increase of the scale factor. It is zero for an object at the origin or in a static universe. Now, consider the second term: the time measured on a clock $(\tau)$ attached to the particle is given by the FRW line element as

$$
\begin{aligned}
c^{2} \mathrm{~d} \tau^{2} & =c^{2} \mathrm{~d} t^{2}-R^{2}(t) \mathrm{d} \chi^{2} \\
\Rightarrow \quad\left(\frac{\mathrm{d} \tau}{\mathrm{d} t}\right)^{2} & =1-\left(\frac{R(t) \dot{\chi}(t)}{c}\right)^{2}
\end{aligned}
$$

Since $\tau$ is observable it must be real (zero for a photon): $(\mathrm{d} \tau)^{2} \geq 0$ implies that $|R(t) \dot{\chi}(t)| \leq c$. Thus, the velocity of the particle due its motion relative to the Hubble flow (or equivalently the homogeneous fluid defining the FRW metric) must be less than the speed of light; its velocity 
due to the increase of the scale factor is not restricted in this way.

We interpret $\dot{R}(t) \chi(t)$ as the increase in distance to the object due to the expansion of the space between the observer and test particle (recession velocity), and $R(t) \dot{\chi}(t)$ as the velocity of object due to its motion through the local rest frame (peculiar velocity). As previously mentioned, we can consider attaching a Minkowski frame to each point in the Hubble flow. Then the speed of light limits the speed of an object through space. But since there is no global Minkowski inertial frame (except for in an empty universe), the relative motion of different regions of the Hubble flow sees no speed limit. Note that the kinematical view sees no difference between recession and peculiar velocities, and thus cannot explain this result. As an illustration, for light moving radially away from the origin: $v_{\mathrm{pec}}=c$, so that $\dot{r}_{\mathrm{p}}=c+H(t) r_{\mathrm{p}}>c$. An observer who insists on extending their Minkowski frame into expanding space will encounter light travelling faster than light!

Note that while the above has ascribed a velocity to be 'due to the expansion of space', we again stress that this is a useful description, rather than a physical cause or law. The physics in operation is general relativity and the ultimate cause of the evolution of the quantities $\left(r_{p}, \dot{r_{p}}, \chi, \dot{\chi}, R, \dot{R}\right)$ is the characteristics (summarised by the equation of state) and initial conditions of the energy in the Universe.

While the picture of expanding space possesses distant observers who are moving superluminally, it is important not to let classical commonsense guide your intuition. This would suggest that if you fired a photon at this distant observer, it could never catch up, but integration of the geodesic equations can reveal otherwise (this is very clear in the conformal representation of FRW universes, see Chodorowski 2006b); this will be examined more deeply in a future contribution. Hence, again, what is important is not the statement of superlumininal motion, but implications for observations, and these must be independent of the framework in which you choose to work.

\subsubsection{Is Everything Expanding?}

An extension of the argument against global expansion given in section 2.2 is that is should be undetectable, since everything will simply expand with it. However, this is not the case: consider a 'normal object', by which we mean one consisting of many particles, held together by internal forces. Suppose that the centre of the object travels along a radial geodesic $\chi_{c}(t)$ in FRW spacetime. Suppose further that the front of the object travels along a trajectory $\chi_{\mathrm{f}}(t)$ that keeps it at a constant proper distance $(L)$ from the centre, i.e.

$$
\begin{aligned}
R(t) \chi_{\mathrm{f}}(t)-R(t) \chi_{c}(t) & =L \quad(\text { a constant }) \\
\Rightarrow \quad \chi_{\mathrm{f}}(t) & =\chi_{c}(t)+\frac{L}{R(t)}
\end{aligned}
$$

The back of the object will move along an analogous path. Then the coordinate trajectory $\chi_{\mathrm{f}}(t)$ is not a geodesic of FRW spacetime. The foremost particle will experience a four-force, which can be calculated by substituting Equation (5) into the equation of motion of a particle experiencing a four-force $f^{a}$ :

$$
\frac{\mathrm{d}^{2} x^{a}}{\mathrm{~d} \lambda^{2}}+\Gamma_{b c}^{a} \frac{\mathrm{d} x^{b}}{\mathrm{~d} \lambda} \frac{\mathrm{d} x^{c}}{\mathrm{~d} \lambda}=\frac{f^{a}}{m}
$$

The observed force in the radial direction is given by projecting $f^{1}$ onto an orthonormal basis; the final result is equation (1) of Harrison (1995) with $U(t)=-H(t) L$ for all time. In the case of $L$ small (compared to $c / H$, the Hubble radius), we have that the radial force $F$ is:

$$
F=-m L \frac{\ddot{R}}{R}
$$

This result tells us how not to understand expanding space. Expanding space does not stretch rigid rulers: how could it? It is just a trick with inertial frames. The internal, interatomic forces in rigid objects are able to maintain the object's dimensions Dicke \& Peebles (1964) argue that EM forces do just this (see also Carrera \& Giulini 2006). Objects are held together by forces that pull their extremities through a succession of rest frames.

It is worth considering what would happen to an object if there were no electromagnetic forces holding it together. Consider an object of many particles with no internal forces. It is shot away from the origin $(\chi=0)$ with speed $v_{0}$, the first particle leaving at time $t_{0}$ and the last at $t_{0}+\Delta t_{0}$. The length of the object is $l_{0}=v_{0} \Delta t_{0}$. The object travels to an observer in the Hubble flow at $\chi$, who measures its speed relative to him $\left(v_{\mathrm{f}}\right)$ and the time of arrival of the first $\left(t_{\mathrm{f}}\right)$ and last particle $\left(t_{\mathrm{f}}+\Delta t_{\mathrm{f}}\right)$ in order to measure its length $\left(l_{\mathrm{f}}=v_{\mathrm{f}} \Delta t_{\mathrm{f}}\right)$. Following Barnes et al. (2006):

$$
\chi=\int_{t_{0}}^{t_{\mathrm{f}}} \frac{\mathrm{d} t}{R\left(1+C_{0} R^{2}\right)^{1 / 2}}=\int_{t_{0}+\Delta t_{0}}^{t_{\mathrm{f}}+\Delta t_{\mathrm{f}}} \frac{\mathrm{d} t}{R\left(1+C_{\mathrm{f}} R^{2}\right)^{1 / 2}}
$$

where $C_{0}$ and $C_{\mathrm{f}}$ are calculated from the initial conditions for each particle. If we assume that $\Delta t_{0}$ and $\Delta t_{\mathrm{f}}$ are small, it follows that we can assume $C_{0}=C_{\mathrm{f}} \equiv C$ and then rearrange the limits of the integral to give ${ }^{4}$

$$
\begin{aligned}
\int_{t_{0}}^{t_{0}+\Delta t_{0}} \frac{\mathrm{d} t}{R\left(1+C R^{2}\right)^{1 / 2}} & =\int_{t_{\mathrm{f}}}^{t_{\mathrm{f}}+\Delta t_{\mathrm{f}}} \frac{\mathrm{d} t}{R\left(1+C R^{2}\right)^{1 / 2}} \\
\Rightarrow \quad \frac{\Delta t_{0}}{R\left(t_{0}\right)\left[1+C R^{2}\left(t_{0}\right)\right]^{1 / 2}} & =\frac{\Delta t_{\mathrm{f}}}{R\left(t_{\mathrm{f}}\right)\left[1+C R^{2}\left(t_{\mathrm{f}}\right)\right]^{1 / 2}}
\end{aligned}
$$

Then, following the method of Barnes et al. (2006) to calculate $v_{\mathrm{f}}=\dot{\chi}\left(t_{\mathrm{f}}\right) R\left(t_{\mathrm{f}}\right)$ and substituting for $C$ we have that

$$
\frac{l_{\mathrm{f}}}{l_{0}}=\frac{v_{\mathrm{f}} \Delta t_{\mathrm{f}}}{v_{0} \Delta t_{0}}=\frac{R\left(t_{\mathrm{f}}\right)}{R\left(t_{0}\right)}
$$

\footnotetext{
${ }^{4}$ This part of the derivation is similar to the derivation of the cosmological redshift directly from the FRW metric, see, among many others, Hobson, Efstathiou, \& Lasenby (2005, p. 368).
} 
Hence, the length of the object has increased in proportion with the scale factor.

This result answers the question: what if an object had no internal forces, leaving it at the mercy of expanding space? This is a rather strange object - it would very quickly be disrupted by the forces of everyday life. Nevertheless, it is a useful thought experiment. The above result shows that the object, being subject only to expanding space, has been stretched in proportion with the scale factor. These are essentially cosmological tidal forces.

We therefore have clear, unambiguous conditions that determine whether an object will be stretched by the expansion of space. Objects will not expand with the universe when there are sufficient internal forces to maintain the dimensions of the object.

\subsubsection{Why Aren't Galaxies or Clusters Pulled Apart by the Expansion of Space?}

Having dealt with objects that are held together by internal forces, we now turn to objects held together by gravitational 'force'. One response to the question of galaxies and expansion is that their self gravity is sufficient to 'overcome' the global expansion. However, this suggests that on the one hand we have the global expansion of space acting as the cause, driving matter apart, and on the other hand we have gravity fighting this expansion. This hybrid explanation treats gravity globally in general relativistic terms and locally as Newtonian, or at best a four force tacked onto the FRW metric. Unsurprisingly then, the resulting picture the student comes away with is somewhat murky and incoherent, with the expansion of the Universe having mystical properties. A clearer explanation is simply that on the scales of galaxies the cosmological principle does not hold, even approximately, and the FRW metric is not valid. The metric of spacetime in the region of a galaxy (if it could be calculated) would look much more Schwarzchildian than FRW like, though the true metric would be some kind of chimera of both. There is no expansion for the galaxy to overcome, since the metric of the local universe has already been altered by the presence of the mass of the galaxy. Treating gravity as a four-force and something that warps spacetime in the one conceptual model is bound to cause student more trouble than the explanation is worth. The expansion of space is global but not universal, since we know the FRW metric is only a large scale approximation.

\subsubsection{The Expansion of Space and Redshift}

The explanation of redshift is a crucial link that needs to be made between cosmological observations and theory. A derivation of the balloon analogy is often employed in the teaching of this concept; a wave is sketched on a balloon and as it is blown up the wavelength is seen to increase as the sketch is stretched along with the expansion of the underlying space. This is largely uncontroversial, but care must be taken in ensuring that the analogy does not mislead. Since we have shown how bodies held together by electromagnetic forces do not expand with the expansion of space, why should electromagnetic waves be affected? The key is to make it clear that cosmological redshift is not, as is often implied, a gradual process caused by the stretching of the space a photon is travelling through. Rather cosmological redshift is caused by the photon being observed in a different frame to that which it is emitted. In this way it is not as dissimilar to a Doppler shift as is often implied. The difference between frames relates to a changing background metric rather than a differing velocity. Page 367 of Hobson, Efstathiou, \& Lasenby (2005) as well as innumerable other texts shows how redshift can be derived very simply by considering the change in the orthonormal basis of observers with different scale factors in their background metrics. This process is discrete, occurring at the point of reception of the photon, rather than being continuous, which would require an integral. If we consider a series of co-moving observers, then they effectively see the wave as being stretched with the scale factor.

\section{Conclusion}

Despite (and perhaps in part because of) its ubiquity, the concept of expanding space has often been articulated poorly and formulated in contradictory ways. That addressing this issue is important must be placed beyond doubt, as the phrase 'expansion of space' is in such a wide use - from technical papers, through to textbooks and material intended for school students or the general public - that it is no exaggeration to label it the most prominent feature of Big Bang cosmologies. In this paper, we have shown how a consistent description of cosmological dynamics emerges from the idea that the expansion of space is neither more nor less than the increase over time of the distance between observers at rest with respect to the cosmic fluid.

This description of the cosmic expansion should be considered a teaching and conceptual aid, rather than a physical theory with an attendant clutch of physical predictions. We have demonstrated the power of this pragmatic conceptualisation in guiding understanding of the universe, particularly in avoiding the traps into which we can be lead without rigorous recourse to general relativity.

The utility of approximation in handling the less tractable properties of cosmologies is undiminished, but the understanding of physical systems therein will be hampered wherever full covariance is absent. All observational properties - whether derived in the dynamically evolving FRW metric or the Minkowski-like conformal representation - must be the same, independent of the choice of co-ordinates. As general relativity approaches its onehundredth birthday, this is a lesson that all cosmologists should learn.

\section{Acknowledgements}

Research undertaken as part of the Commonwealth Cosmology Initiative (CCI: www.thecci.org), an international collaboration supported by the Australian Research Council. L.A.B. and J.B.J. are supported by an Australian 
Postgraduate Award. L.A.B. is also supported by a University of Sydney School of Physics Denison Merit Award. J.B.J. is also generously supported by the University of Edinburgh. M.J.F. is supported by a University of Sydney Faculty of Science UPA scholarship. G.F.L. gratefully acknowledges support through the University of Cambridge Institute of Astronomy visitor program. Brendon Brewer is thanked for helping suggest the title. This work has been supported by the Australian Research Council under grant DP 0665574.

\section{References}

Abramowicz, M. A., Bajtlik, S., Lasota, J.-P. \& Moudens, A., 2006 ArXiv e-prints, astro-ph/0612155

Barnes, L., Francis, M. J., James, J. B. \& Lewis, G. F., 2006, MNRAS, 373, 382

Carrera, M. \& Giulini, D., 2006 (astro-ph/0602098)

Chodorowski, M., 2006, Concepts Phys, 4, 17
Chodorowski, M., 2006, MNRAS, 378, 239

Davis, T. M. \& Lineweaver, C. H., 2004, PASA, 21, 97

Davis, T. M., Lineweaver, C. H. \& Webb, J. K., 2001, Am. J. Phys., 71,358

Dicke, R. H. \& Peebles, P. J., 1964, PhRvL, 12, 435

Grøn, O. \& Elgarøy, O., 2006, Am. J. Phys., in press (astro$\mathrm{ph} / 0603162)$

Harrison, E. R., 1995, ApJ, 446, 63

Harrison, E., 2000, 'Cosmology: The Science of the Universe' (Cambridge, UK: Cambridge University Press)

Hobson, M. P., Efstathiou, G. P. \& Lasenby, A. N., 2005, 'General Relativity: An Introduction for Physicists' (Cambridge, UK: Cambridge University Press)

Lieu, R., 2007, ArXiv e-prints, astro-ph:0705.2462

Page, D., 1993, ArXiv e-prints, gr-qc:9303008

Rees, M. J. \& Weinberg, S., 1993, New Scientist, 1869, 32

Tipler, F. J., 1996, MNRAS, 282, 206

Whiting, A. B., 2004, Obs, 124, 174

Weinberg, S., 1972, Gravitation and Cosmology: Principles and Applications of the General Theory of Relativity (Wiley-VCH) 